\title{
A 50 Year-Old Homeless Man With Symptomatic Palpitations
}

Neerav G. Sheth MD, Daniel R. Frisch, MD

\section{Case Report}

The patient is a 50 -year old man with Hepatitis C, HIV, and pneumonia who recently became homeless and presented with 3 days of worsening palpitations and shortness of breath. Initially the palpitations were sporadic; however, they had become more frequent over the three days prior to admission. He had a corresponding increase in shortness of breath along with significant decreases in his exercise tolerance. He presented to the Emergency Department (ED) when he could no long perform his usual activities of daily living. In the ED, patient was found to have a narrow complex tachycardia with a rate of 234 beats per minute (bpm) (Figure 1).

His past history is significant for HIV diagnosed in 1979 with a recent CD4 Count of 16 . The patient was not on HAART medication due to noncompliance resulting from financial issues. He also has a history of Hepatitis C secondary to remote intravenous drug abuse. He had pneumonia approximately three weeks prior to admission, which was incompletely treated with antibiotics. The patient also noted a 30-pack year history of tobacco use.

In the $\mathrm{ED}$, the patient was given large volumes of normal saline followed by 3 sequential doses of adenosine, all of which failed to terminate his tachycardia. He was then started on an esmolol infusion with resultant hypotension to a systolic blood pressure (SBP) of $80 \mathrm{mmHg}$. The esmolol infusion was subsequently stopped and patient received diltiazem. Following the diltiazem infusion, the patient still had no decrease in his rapid heart rate. A cardiology consultation was requested and procainamide was recommended for chemical cardioversion; however, the patient's blood pressure had deteriorated to SBP of $70 \mathrm{mmHg}$ with a heart rate of $240 \mathrm{bpm}$ and the patient was urgently electrically cardioverted. Sinus rhythm was restored at a rate of $110 \mathrm{bpm}$ and the SBP improved to $130 \mathrm{mmHg}$. He was then referred to the Electrophysiology (EP) service in order to evaluate for a more durable and definitive management of his supraventricular tachycardia (SVT). An EP study was performed and revealed an atrioventricular accessory pathway on the left lateral mitral annulus that was responsible for his SVT. The accessory pathway was successfully ablated as evidenced by intracardiac electrograms and by a lack of initiation of supraventricular tachycardia following isoproteronol administration after ablation (Figure 2).

\section{Discussion}

Supraventricular tachycardia (SVT) is an accelerated rhythm occurring with a prevalence of $2.25 / 1000$ in the general population with an incidence of $35 / 100,000$ person-years. ${ }^{1}$ The three most common mechanisms of SVT are atrioventricular nodal reentrant tachycardia (AVNRT), atrioventricular reentrant tachycardia (AVRT), and atrial tachycardia (AT). A common feature of all three mechanisms is a narrow QRS complex (usually identical to the QRS seen in sinus rhythm), which distinguishes these tachycardias from ventricular

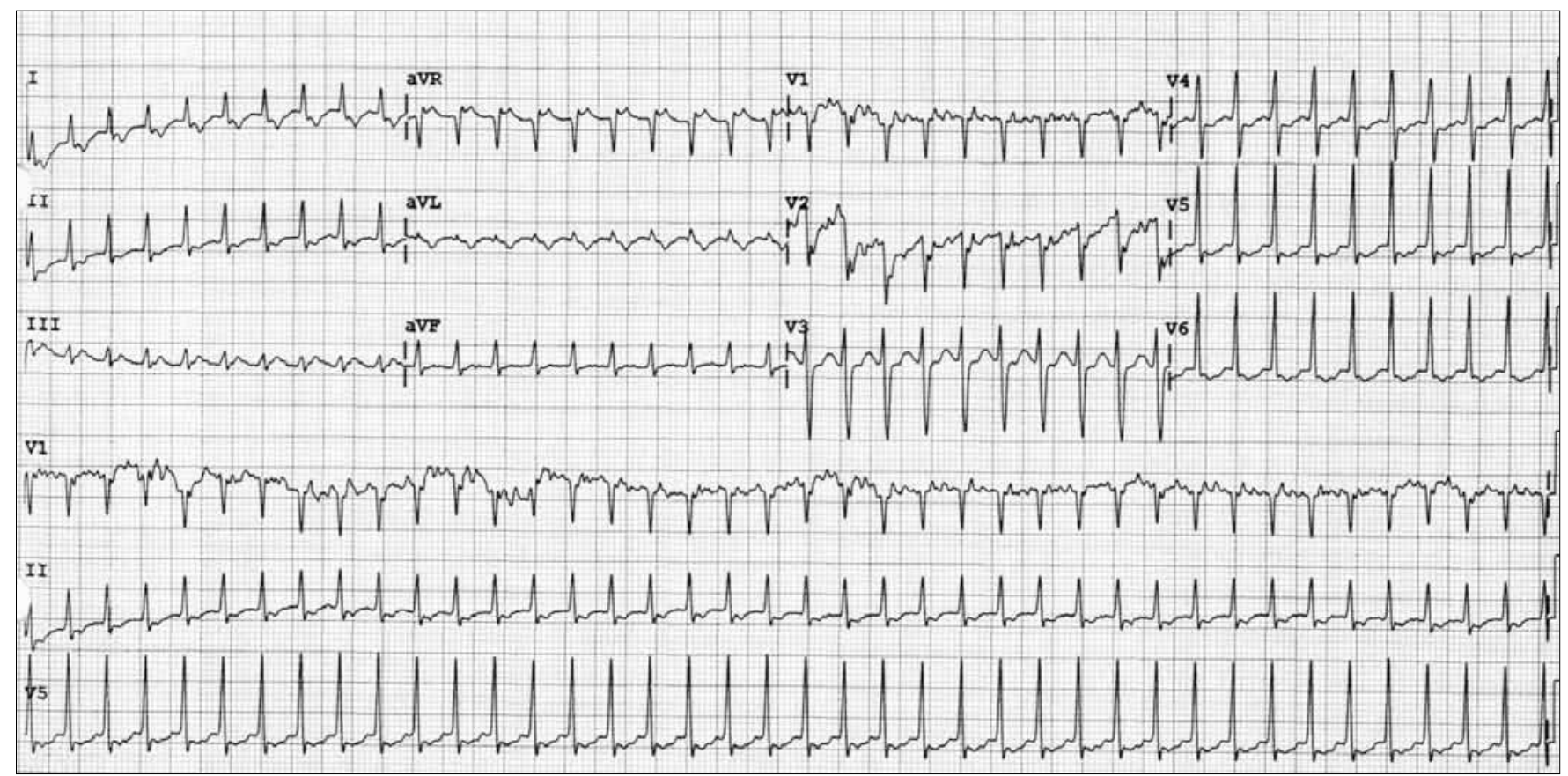

Figure 1. Supraventricular Tachycardia on admission ECG 


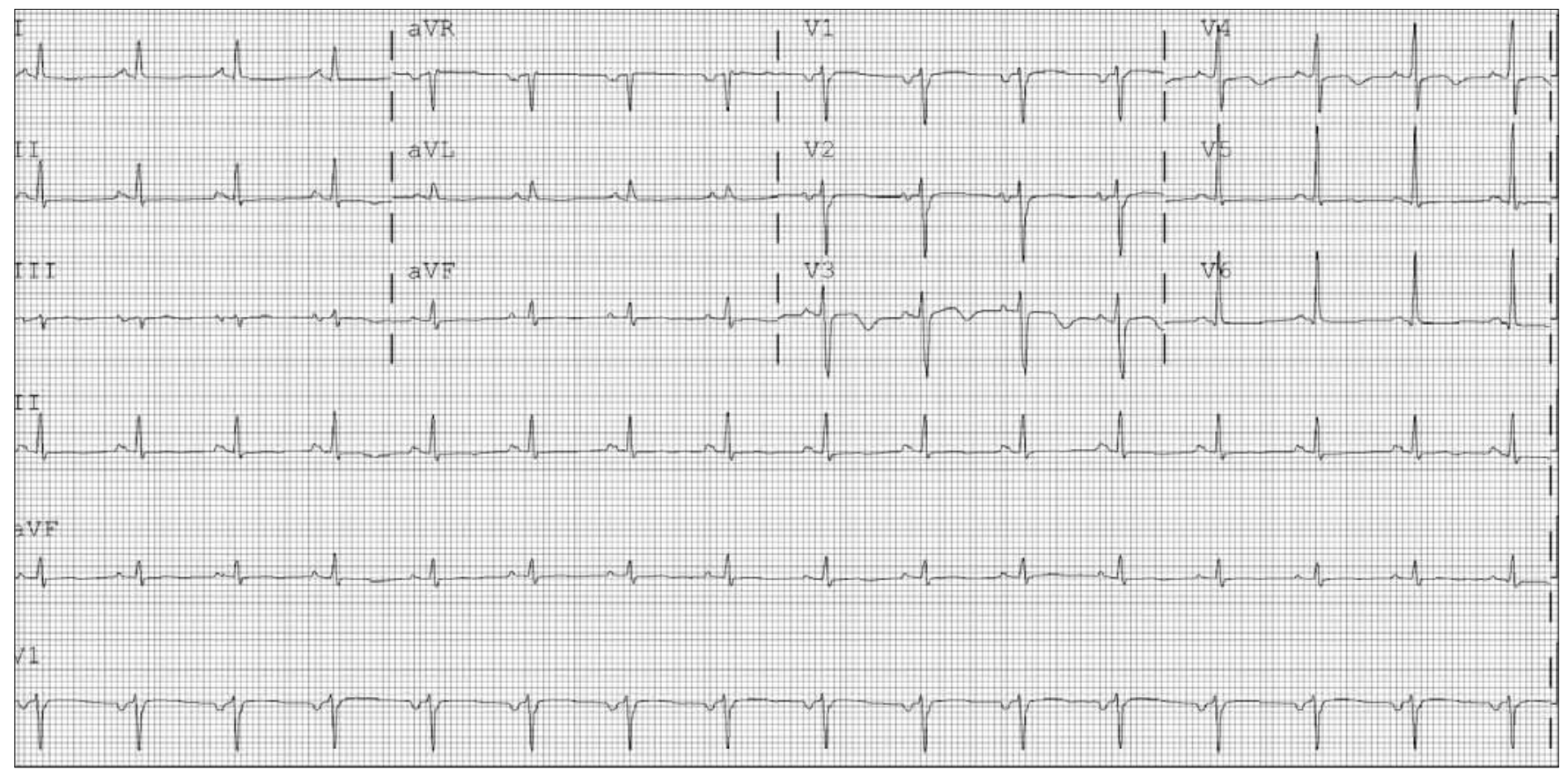

Figure 2. Post Catheter Ablation ECG

tachycardia. Of the three types, AVNRT is the most common, followed by AVRT. Women more often present with AVNRT, while men, particularly younger men, are more likely to present with AVRT. ${ }^{2}$ AT is more likely to present in patients with advanced age and/or structural heart disease. While a surface electrocardiogram can often diagnose which of the three mechanisms is present, ${ }^{3}$ an EP study is the gold standard for diagnosis.

A spectrum of strategies exists for the initial management of SVT. These include invasive EP studies (i.e. catheter ablation), noninvasive medical management, and even watchful waiting. Typically medications (beta blockers, calcium channel blockers) are used to control the heart rate in SVT. In rare circumstances, anti-arrhythmic medications such as digoxin and procainamide can be used as well. However, in all instances of proven SVT, an EP study with catheter ablation should be considered as a first-line treatment (ACC/AHA/ESC Guidelines Class I, Level of Evidence $\mathrm{B}$ recommendation). ${ }^{4}$ This is true even for a patient's initial presentation of SVT.

In this case, the patient's initial presentation of SVT was highly symptomatic and required urgent care for stabilization and an expedited EP study with catheter ablation for definitive treatment. Though it may seem aggressive, this was likely the best option for this patient. He had been previously non-compliant with prescribed medications and had an inability to follow up regularly with physicians, likely due to socioeconomic hardship. The dilemma posed here is how aggressively to treat this patient's first documented episode of SVT. Despite the fact that EP studies are invasive and expensive procedures, frequently an EP study may be more clinically beneficial as well as cost effective. A single treatment that provides durable benefit is appealing and is likely to decrease a patient's burden of disease and health care utilization for this issue. While ethically we are obligated as physicians to do what is in the best interest of the patient without regard to socioeconomic status, we are not immune from the economic reality of the price tag associated with our treatments. The initial investment of a relatively aggressive procedure in this case accomplished the goal of resolving this patient's symptoms and addressing the challenge of medical compliance.

\section{References}

1. Orejarena LA, Vidaillet H, Jr., DeStefano F, et al. Paroxysmal supraventricular tachycardia in the general population. J Am Coll Cardiol 1998;31:150 -7.

2. Huang SKS, Wood M, eds. Catheter Ablation of Cardiac Arrhythmias. Philadelphia, PA: Saunders Elsevier; 2006: 473-490.

3. Kumar Uday, Rao Rajni, Scheinman Melvin. The 12-Lead Electrocardiogram in Supraventricular Tachycardia. Cardiol Clinics 2006;24:427-437.

4. Blomström-Lundqvist et al., Management of Patients with Supraventricular Arrhythmias. ACC/AHA/ESC Guidelines for the Management of Patients With Supraventricular Arrhythmias: A Report of the American College of Cardiology/American Heart Association Task Force on Practice Guidelines and the European Society of Cardiology Committee for Practice Guidelines (Writing Committee to Develop Guidelines for the Management of Patients With Supraventricular Arrhythmias) J Am Coll Cardiol 2003;42:1493-531. 